\title{
Non Alcoholic Fatty Liver Disease- Is It Always Benign?
}

\author{
M RAHMAN ${ }^{a}$, T ABEDIN $^{b}$, RODED AMIN ${ }^{c}$, MR RAHMAN ${ }^{d}$, MA FAIZ ${ }^{\mathrm{e}}$
}

\begin{abstract}
Summary:
Nonalcoholic fatty liver disease (NAFLD) is the most common liver disease observed in the clinical practice of hepatology affecting approximately $20 \%$ of the general population. It is increasingly apparent that non alcoholic steatohepatits (NASH) and NAFLD are not Western disease. There is evolution of Western-style life among the Asian population and NASH has increasingly been diagnosed in several regions in Asia. NASH is considered as a type of a larger spectrum of NAFLD that is a consequence of insulin resistance and other underlying factors with histological findings ranging from fatty change alone to fat plus inflammation, to fat plus ballooning degeneration, and to fat plus alcoholic hepatitis-like lesions including Mallory body and fibrosis, the latter two categories being considered as NASH. Although liver biopsy is currently the gold standard for diagnosis, there is a need for less invasive methods. Imaging by ultrasound, computerized tomography and magnetic resonance are all able to demonstrate fat. Ultrasound, although probably not the most reliable imaging method, has many advantages and, when positive, gives a
\end{abstract}

Introduction:

Nonalcoholic steatohepatitis (NASH) represents a part of a wide spectrum of non-alcoholic fatty liver disease (NAFLD), which ranges from simple

a. Dr. Mohammad Mohibur Rahman, MBBS, FCPS (Internal Medicine), MD (Gastroenterology), Assistant Professor, Department of Gastroenterology, Shahabuddin Medical College, Gulshan-2, Dhaka.

b. Dr Tariq Abedin, MBBS, Department of Medicine, Bangabandhu Sheikh Mujib Medical University, Dhaka.

c. Dr. Robed Amin, MBBS, FCPS (Internal Medicine), Junior consultant (Medicine), Upozilla Health complex, Hathazari, Chittagong.

d. Professor M Ridwanur Rahman, MBBS, FCPS (Internal Medicine), Professor of Medicine, Begum Khaleda Zia Medical College, Dhaka

e. Professor Md Abul Faiz, MBBS, PhD, FCPS (Internal Medicine), FRCP, Principal and Head of the Department of Medicine, Dhaka Medical College, Dhaka.

Address of Correspondence: Dr. Mohammad Mohibur Rahman, Assistant Professor, Department of Gastroenterology, Shahabuddin Medical College, House no 15-16, Road 113/A, Gulshan-2, Dhaka1212. E-mail: jimad_2003@yahoo.com.

Received: 15 January, 2007

Accepted: 26 July, 2007 high degree of certainty of the diagnosis depending on the prevalence of fatty liver in the population being studied. Unlike liver biopsy, none of these techniques is able to differentiate simple steatosis from non- alcoholic steatohepatitis. The ultimate goal of treating the patient with NASH is to prolong life by avoiding the end-organ diseases associated with insulin resistance and the metabolic syndrome. Treatment of patients with nonalcoholic fatty liver has typically been focused on the management of associated conditions as well as discontinuation of potentially hepatotoxic drugs. Weight loss and exercise improve insulin sensitivity. Bariatric surgery may improve liver histology in patients with morbid obesity. Insulin sensitising drugs showed promise in pilot trials as have a number of hepatoprotective agents. Further randomised, well controlled trials are required to determine the efficacy of these drugs.

In this article, we will review (1) various processes that are involved in the pathogenesis of NASH (2) the existing medical therapy for patients with nonalcoholic fatty liver, (2) the emerging and potentially useful medications.

(J Bangladesh Coll Phys Surg 2007; 25 : 144-152)

steatosis and steatohepatitis to advanced fibrosis and cirrhosis. ${ }^{1}$ Nonalcoholic fatty liver disease (NAFLD) is the most common liver disease observed in the clinical practice of hepatology. ${ }^{2}$ NASH today is third most common cause of chronic liver disease in North America after alcoholics liver disease and hepatitis and the most common cause of raised transaminases more than six-months. Primary NAFLD is related to insulin resistance and thus frequently occurs as part of the metabolic changes that accompany obesity, diabetes and hyperlipidaemia. However, it is important to exclude secondary causes of hepatic steatosis by clinical assessment. Treatment of these conditions differs and revolves around correcting the underlying cause. ${ }^{3}$

\section{Methodology:}

PubMed was searched in June 2006 for all Englishlanguage publications including the search terms "Non alcoholic fatty liver disease", "Non alcoholic steatohepatitis" from 1980 onwards. The full articles for selected identified records that were thought to be 
potentially relevant were obtained. The references in these selected articles were also checked for additional information that might be relevant.

Definition: Non-alcoholic fatty liver disease (NAFLD) represents a spectrum of liver diseases characterized mainly by macrovesicular steatosis that occurs in the absence of alcohol consumption in amounts considered injurious to the liver. ${ }^{4}$ The hepatic histology can vary from isolated hepatic steatosis alone to steatohepatitis and are referred to as non-alcoholic fatty liver (NAFLD) and non-alcoholic steatohepatitis (NASH) respectively. In addition to predominantly macrovesicular steatosis, the diagnosis of steatohepatitis also requires the additional presence of varying combinations of findings including cytologic ballooning, Mallory's hyaline, scattered inflammation and pericellular fibrosis. ${ }^{4} \mathrm{NASH}$ is may be defined as a syndrome characterized by the association of fatty liver and lobular hepatitis and chronically elevated ALT plasma levels in patients with negligible alcohol intake. ${ }^{5}$ The syndrome is mainly associated with the metabolic syndrome which is characterized by a constellation of findings including obesity, diabetes, hypertension and hypertriglyceridemia obesity, diabetes and dyslipidaemia. ${ }^{4,6}$ But a few patients are lean, have normal fasting glucose and glucose tolerance, and show no evidence of increased plasma lipids. Patients with fatty liver and hepatitis are identified as having NASH. ${ }^{6}$

Epidemiology: The last two or three decades have seen the evolution of Western-style life of near complete inactivity, energy-dense food choices and liberal fiscal resources to obtain them and other means to avoid physical activity. Moreover, what is increasingly apparent is that NASH and NAFLD is not a Western disease and many population groups in the Asia-Pacific region are particularly prone to type 2 diabetes. For this reason, NASH has increasingly been diagnosed in several regions in Asia including Indonesia, Malaysia, Philippines, Thailand and India. $^{7}$

True prevalence of NASH is difficult to assess without large-scale epidemiological studies. Steatosis is the most common cause of raised transaminases and affects nearly $10-24 \%$ of general population. 8,9
In india, only $2-3 \%$ in the general population have steatosis.

The most important risk factors for NAFLD are obesity, diabetes, hyperlipidemia and female sex. Prevalence of obesity in non alcoholic steatohepatitis ranges from $30-100 \%$ in various series of patients. NAFLD may occur in up to $75 \%$ of type 2 diabetes, although obesity may be a confounding variable. $1,4,10$

\section{Causes of non-alcoholic fatty liver disease}

Primary:

Obesity, glucose intolerance, hypertriglyceridaemia, low HDL cholesterol, hypertension.

Secondary:

Nutritional

Protein-calorie malnutrition, rapid weight loss, gastrointestinal bypass surgery, total parental nutrition, short bowel syndrome, small bowel bacterial overgrowth, e.g. small bowel diverticulosis.

Drugs

Glucocorticoids, oestrogens, tamoxifen, amiodarone, methotrexete, diltiazem, zidovudine, valproate, aspirin, tetracycline, cocaine, protease inhibitors.

Metabolic

Lipodystrophy, hypopituitarism, dysbetalipoproteinaemia, Weber-Christian disease. Lipid loss versus lipid deposition syndromes,

Toxins

Amanita phalloides mushroom, phosphorus poisoning, petrochemicals, bacillus cereus toxin.

Infections

Human immunodeficiency virus, hepatitis C.

Others

Chronic inflammatory disorders, e.g. rheumatoid arthritis and systemic lupus erythematosus.

Pathogenesis (figure I):

The pathogenesis of NAFLD is not fully understood, however the finding that not all patients with steatosis develop hepatic inflammation and hepatocellular damage has led to the hypothesis that different pathogenic factors lead firstly to hepatic steatosis and secondly to hepatic damage ("the second hit"). ${ }^{10}$ Accumulation of hepatic fat is closely linked to 
insulin resistance. ${ }^{11}$ Insulin resistance is present in approximately $98 \%$ of individuals with NAFLD and over $80 \%$ of subjects with NAFLD meet minimal criteria for the metabolic syndrome. ${ }^{4}$ Insulin resistance which increases lipolysis of peripheral adipose tissue with resultant increased fat influx into the liver in the form of free fatty acids. Insulin resistance also promotes de novo triglyceride synthesis within the liver and inhibits fatty acid oxidation thereby promoting triglyceride accumulation. ${ }^{11}$ It is unknown what "second hit" leads to the development of liver damage, although several factors have been implicated including oxidative stress, mitochondrial abnormalities, and hormonal disturbances involving leptin and adiponectin. ${ }^{12}$

The recently discovered hormone resistin is linked to the development of insulin resistance, but direct evidence of resistin levels in humans with nonalcoholic fatty liver disease (NAFLD) is lacking. One study showed that NAFLD patients have increased circulating resistin and that increased levels are not correlated to either insulin resistance or BMI, but only to the histological severity of the disease. ${ }^{13}$

Figure I: Metabolic alterations resulting in hepatic triglyceride accumulation in insulin-resistant states. ${ }^{11}$

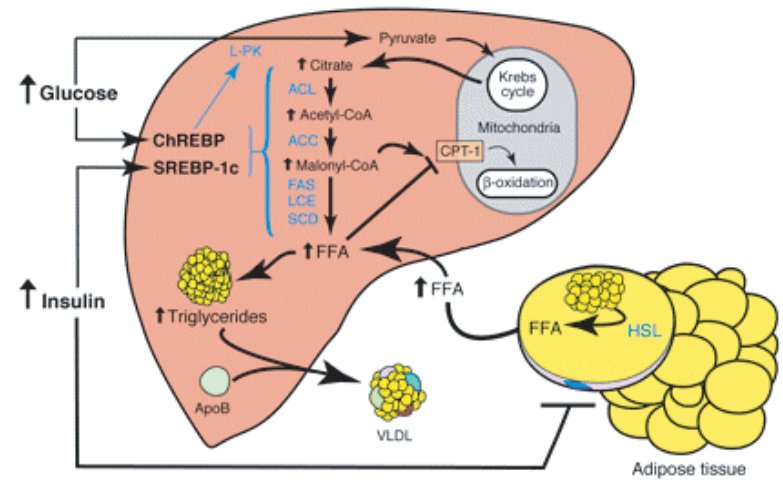

(Note: FFA: Free Fatty acid, HSL: hormone-sensitive lipase FAS: fatty acid synthase LCE: long-chain fatty acyl elongase, SCD: stearoyl-CoA desaturase, ACC: Acetyl-CoA carboxylase, Ch REBP: carbohydrate response element binding protein SREBP-1c: Sterol regulatory element-binding protein-1c, ApoB: CPT1: carnitine palmitoyl transferase-1).
Natural history

NAFLD exists as a histological spectrum of changes; simple steatosis refers to $>5 \%$ hepatic steatosis in the absence of significant inflammation and hepatocellular damage whereas NASH demonstrates inflammation and hepatocellular damage and sometimes fibrosis. ${ }^{14}$ NAFLD may be progressive resulting in cirrhosis that may be complicated by hepatocellular carcinoma and liver failure. Overall, about $5 \%$ of patients with NAFLD develop cirrhosis over an average of a seven year period with $1.7 \%$ dying from complications of liver cirrhosis. ${ }^{15}$

The high prevalence and chronic nature NAFLD subsequently translates to a significant health burden for the general community. In addition, subjects with a diagnosis of NAFLD have a higher risk of all cause mortality than the general population. ${ }^{10}$ Many patients with nonalcoholic fatty liver disease have a relatively benign course, whereas in some others, the disease progresses to cirrhosis and its complications. ${ }^{3}$ Histological features assist in stratifying patient risk of progressive liver disease. ${ }^{10}$ Patients found to have pure steatosis on liver biopsy seem to have the best prognosis within the spectrum of nonalcoholic fatty liver disease, whereas features of steatohepatitis or more advanced fibrosis are associated with a worse prognosis. ${ }^{3}$ Simple steatosis is comparatively benign with a $0 \%-4 \%$ risk of developing cirrhosis over a one to two decade period. In contrast, $5 \%-8 \%$ of patients with NASH may develop cirrhosis over approximately five years. Assessment of fibrosis stage is also valuable in prognosticating risk of developing liver related morbidity, with patients with advanced fibrosis (bridging fibrosis and cirrhosis) at most risk. Although these features aid in stratifying patients at risk, a significant proportion of patients will have all of these adverse prognostic markers but will not develop liver related morbidity or mortality. Thus accurate prediction of those patients who will benefit most from treatment is difficult. ${ }^{10}$

\section{Clinical Features}

Most patients with nonalcoholic fatty liver disease have no symptoms or signs of liver disease at the time of diagnosis. ${ }^{3}$ Commonest symptoms are fatigue or malaise and a sensation of fullness or discomfort on the right side of the upper abdomen. ${ }^{3,4}$ Hepatomegaly is the only physical finding in most patients. ${ }^{3}$ Other 
features are pruritus, oedema, stigmata of chronic liver disease, acanthosis nigricans, obesity, diabetes, hypertension, dyslipidemia. ${ }^{3,4}$

\section{Diagnosis of Nonalcoholic Fatty Liver Disease Presumptive Diagnosis: ${ }^{21}$}

Elevated serum liver enzyme levels (AST, ALT, or glutamyl-transferase) or imaging study with evidence of fat and minimal or no alcohol intake and negative test results for viral hepatitis, autoimmune disease (primary biliary cirrhosis), and congenital liver disease (e.g, Wilson disease). Definitive Diagnosis: Liver biopsy specimen with evidence of fat with or without inflammation or fibrosis and minimal or no alcohol intake.

The diagnosis of NASH can be done on the basis of the following criteria: ${ }^{19}$

(1) Intake of less than $20 \mathrm{~g}$ of ethanol per day,

(2) Biopsy proven steatohepatitis; steatosis, inflammatory infiltrates, and ballooning degeneration with or without Mallory bodies or pericellular/ perivenular fibrosis,

(3) Appropriate exclusion of other liver diseases.

\section{Identify cause of NAFLD}

In the presence of features of the metabolic syndrome, further evaluation of other causes for NAFLD is usually unnecessary. But detailed drug history is mandatory in all cases. The initial clinical evaluation should include an assessment of adipose tissue distribution to look for lipodystrophy. The initial laboratory evaluation should include a fasting lipid profile. ${ }^{4}$

\section{Laboratory abnormalities:}

Mildly to moderately elevated serum levels of aspartate aminotransferase, alanine aminotransferase (Usually $<250 \mathrm{u} / 1$, or both are the most common and often the only laboratory abnormality found in patients with nonalcoholic fatty liver disease. ${ }^{3,4}$ The ratio of aspartate aminotransferase to alanine aminotransferase is usually less than 1 , but this ratio increases as fibrosis advances. ${ }^{3}$ Other abnormalities, including hypoalbuminemia, a prolonged prothrombin time, and hyperbilirubinemia, may be found in patients with cirrhotic-stage nonalcoholic fatty liver disease. Elevated serum ferritin levels are found in half the patients and increased transferrin saturation is found in 6 to 11 percent of patients. ${ }^{3}$

Imaging studies:

The diagnosis of NAFLD requires confirmation of hepatic steatosis by imaging or liver biopsy with clinical exclusion of excessive ( $>20 \mathrm{~g} /$ day) alcohol ingestion. ${ }^{14}$ Ultrasound, computed tomography, or magnetic resonance studies can confirm the presence of hepatic steatosis with a comparatively high degree of accuracy. ${ }^{16,17}$ The presence of $>33 \%$ fat on liver biopsy is optimum for detecting steatosis on radiological imaging. Radiological modalities are unable to distinguish between NASH and other forms of NAFLD, a distinction that has important prognostic implications. ${ }^{17}$

Ultrasound is comparatively cheap and readily available but is less sensitive at detecting minimal $(<30 \%)$ steatosis or among obese patients (BMI of $\left.35-40 \mathrm{~kg} / \mathrm{m}^{2}\right) .{ }^{18}$ Thus a negative ultrasound does not necessarily exclude NAFLD. The sonographic features of NAFLD include increased hepatic parenchymal echotexture and vascular blurring. These findings are however also seen in those with any form of chronic liver disease and, although sensitive (85-95\%), they are non-specific (positive predictive value $62 \%) .{ }^{4}$ The severity of hepatic echogenicity is graded as follows:

Grade 0: normal echogenicity, grade I, slight diffuse increase in fine echoes in liver parenchyma with normal visualization of the diaphragm and intra hepatic vessel borders; grade 2, moderate, diffuse increase in fine echoes with slightly impaired visualization of intra hepatic vessels and diaphragm; grade 3, marked increase in fine echoes with poor or non-visualization of intra hepatic vessels borders, diaphragm and posterior right lobe of the liver. ${ }^{17}$

CT imaging: CT imaging of the liver provides a more specific method for the non-invasive diagnosis of NAFLD. Hepatic steatosis decreases the CT attenuation of the liver. While these features allow hepatic steatosis to be defined with a $76 \%$ positive predictive value 4 The severity of hepatic fatty infiltration is graded as follows: grade 0, normal; grade 1, liver attenuation slightly less than spleen; grade 2, more pronounced difference between liver and spleen and intra hepatic vessels not seen or 
slightly higher attenuation than liver; grade 3, markedly reduced liver attenuation with sharp contrast between liver and intra hepatic vessels. ${ }^{17}$

MRI: MRI is even more sensitive than a CT scan for the assessment of hepatic steatosis ${ }^{4}$ Despite minimal improvement in the interobserver concordance, MRI does not offer any additional advantages over CT or ultrasonogram in detecting the pathologic features that are important in establishing the diagnosis of NASH. The minimal advantages of MRI should be balanced against the wider availability and lower cost of ultrasonogram. ${ }^{17}$

Given the modest increase in diagnostic accuracy and marked increase in cost with CT imaging and MRI, a hepatic sonogram is the most commonly used imaging modality to diagnose a fatty liver. However, it is important to note that none of these methods can diagnose steatohepatitis or accurately assess the stage of the disease. ${ }^{4}$

\section{Liver biopsy:}

The detection of NASH is usually delayed and there are no serum surrogate markers for NASH, ${ }^{19}$ Hence the precise diagnosis of NAFLD and the distinction between hepatic steatosis and steatohepatitis requires a liver biopsy. ${ }^{4,} 19$ The presence of severe fibrosis, the most worrisome feature in liver biopsies in patients with NASH, has been noted in 15 to $50 \%$ of patients, whereas well established cirrhosis has been documented in $7 \%$ to $26 \%$ of patients at the time of diagnosis. ${ }^{20}$

So Liver biopsy is the gold standard for diagnosis and is the only investigation able to distinguish between simple steatosis and NASH or stage the degree of fibrosis. ${ }^{17}$ Liver biopsy remains not only the best diagnostic tool for confirming NASH, but also the most sensitive and specific means of providing important prognostic information in patients with this condition. ${ }^{20}$

Given the large number of afflicted subjects, the invasive nature of a liver biopsy and the lack of effective treatment, there is often a reluctance to pursue this diagnosis with vigour and many patients are left with a diagnosis of suspected NAFLD. The need to perform a biopsy and make these distinctions continue to generate controversy. ${ }^{4}$ There are three basic principles that determine the need and the aggressiveness with which one should try to evaluate any medical problem. First, one must consider the differential diagnosis and the potential possibilities that exist. Next, one must know the natural history of these conditions and consider which diagnoses, if missed, will have dire consequences for the individual patient. The answer to this question is linked to the critical final question: even if the diagnosis is made, is effective treatment available? ${ }^{4}$

The decision to perform a liver biopsy must be individualised and may be useful when there is diagnostic uncertainty or to provide prognostication regarding outcome. The clinical corollary is that individuals without risk factors for NAFLD are more likely to have an alternate cause for their abnormal liver enzymes. ${ }^{4}$ Liver biopsy may also be performed in patients with risk factors of advanced fibrosis (diabetes, obesity, age $>45$, AST: ALT $>1$ ), ${ }^{20}$ where a diagnosis of cirrhosis has implications for screening for varices and hepatocellular carcinoma. ${ }^{10}$

\section{Importance of NASH:}

NASH progresses to cirrhosis at a rate that is generally similar to other causes of chronic hepatitis. Therefore, conservative extrapolation from the NHANES III data suggests that 6.4 million US adults have NAFLD (69\% of the 9.1 million individuals with cryptogenic aminotransferase level elevations), and 640000 of these may have cirrhosis (assuming that cirrhosis develops in $10 \%$ of those with NASH). In comparison, population-based studies suggest that less than $2 \%$ of the US adult population ( 2 million individuals) are infected with chronic hepatitis C. If $20 \%$ of the chronic hepatitis $\mathrm{C}$ population develops cirrhosis, then chronic hepatitis $\mathrm{C}$ accounts for approximately 500000 cases of cirrhosis, fewer than caused by NASH. ${ }^{21}$ But compensated cirrhosis due to NASH is associated with a lower mortality rate compared with that due to HCV. It is also associated with a lower rate of development of ascites, hyperbilirubinemia, and hepatocellular carcinoma. ${ }^{22}$ NAFLD is significantly associated with a moderately increased CVD risk among type 2 diabetic individuals. This relationship is independent of classical risk factors and is only partly explained by occurrence of metabolic syndrome. ${ }^{23}$ 
NAFLD and hepatitis $\mathrm{C}$-risk factors and clinical implications:

Hepatitis C and NAFLD are the two most common liver diseases in the Western hemisphere. It is therefore natural that these conditions often co-exist in the same individual. Hepatitis C, especially genotype 3 , is often associated with hepatic steatosis. ${ }^{29}$ Steatosis influences chronic hepatitis C progression in a genotype specific way. ${ }^{30}$ In subjects with genotype 3 infection, a sustained virologic response to treatment is associated with improvement in hepatic steatosis. The diagnosis of NAFLD in a subject with hepatitis $\mathrm{C}$ infection is based on the presence of hepatic steatosis. The presence of NAFLD in subjects with hepatitis C genotype 1 infection is most strongly associated with the presence of the metabolic syndrome and insulin resistance. The degree of hepatic steatosis correlates with the degree of hepatic fibrosis and the presence of concomitant steatosis is associated with more advanced fibrosis. Insulin resistance and hyperinsulinemia have been associated with increased collagen production by hepatic stellate cells. Subjects with hepatitis C and NAFLD are more likely to be virologic nonresponders following anti$\mathrm{HCV}$ therapy. The value of treating insulin resistance and NAFLD prior to antiviral therapy remains to be experimentally verified. ${ }^{29}$

Treatment (Figure II):

There is currently no proven therapy for NASH. ${ }^{21}$ Treatment strategies for NAFLD have revolved around (1) identification and treatment of associated metabolic conditions such as diabetes and hyperlipidaemia; (2) improving insulin resistance by weight loss, exercise, or pharmacotherapy; (3) using hepato-protective agents such as antioxidants to protect the liver from secondary insults. ${ }^{10,24}$

Figure II Treatment algorithm for NAFLD ${ }^{10}$

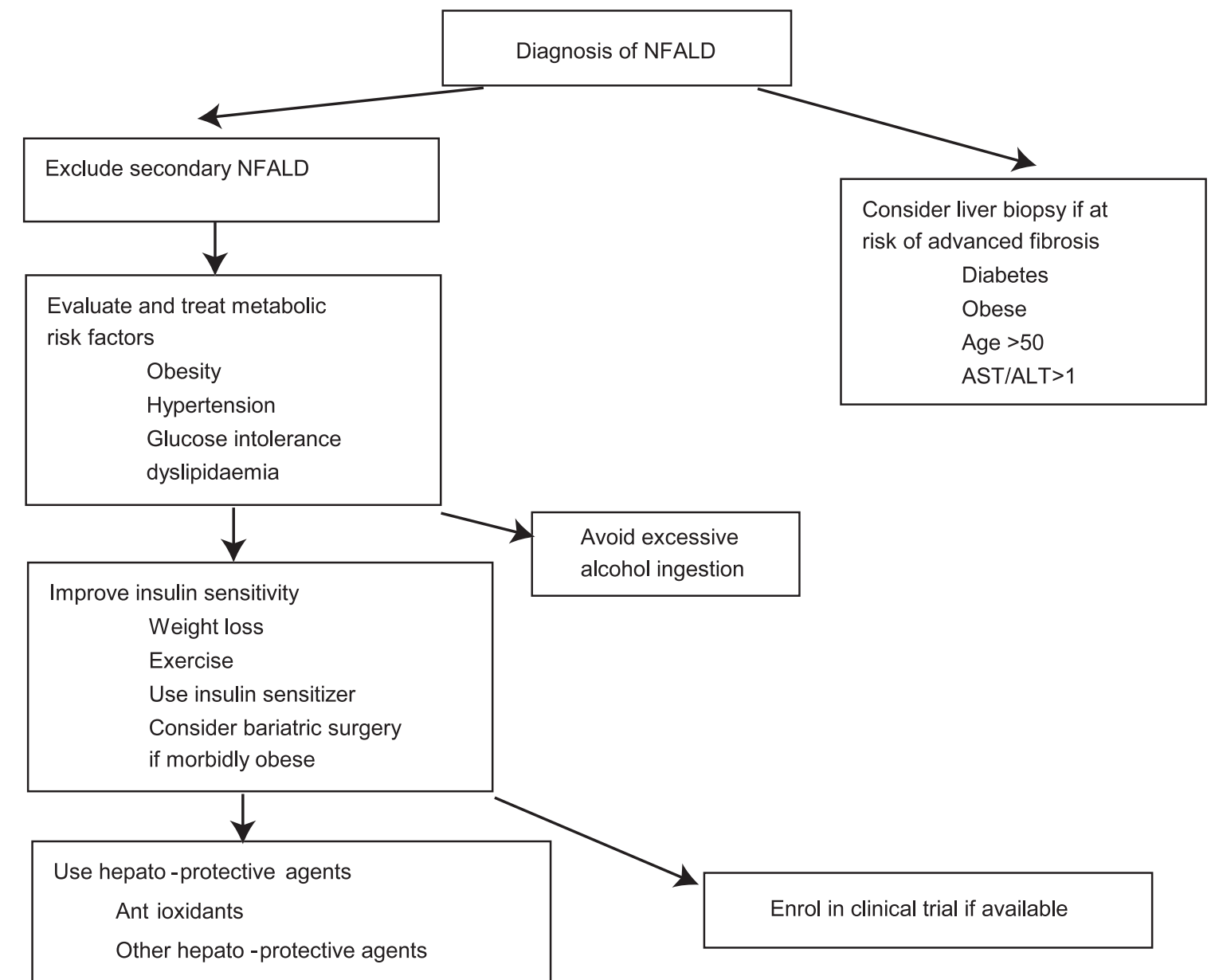


The goals of treatment include (1) correction of the underlying risk factors, (2) avoidance of factors that promote progression of liver disease, and (3) specific treatment of non-alcoholic steatohepatitis. ${ }^{25}$

Treatment of associated metabolic conditions:

The features of metabolic syndrome are commonly present in subjects with NAFLD, with $67 \%-71 \%$ being obese, $12 \%-37 \%$ having impaired fasting glycaemia, $57 \%-68 \%$ having disturbed lipid profiles, and $36 \%-70 \%$ being hypertensive. Therefore, patients with newly diagnosed NAFLD should be screened for these conditions and appropriate treatment instituted in an effort to ameliorate the vascular risk as well as to improve NAFLD. ${ }^{10}$ The ultimate goal of treating the individual is to prolong life by avoiding the end-organ diseases associated with insulin resistance and the metabolic syndrome. Thus, effective treatment of the metabolic syndrome is mandatory in patients with NAFLD and insulin resistance on general principles. ${ }^{4}$

\section{Weight loss and exercise:}

The most common risk factor for NASH is obesity. ${ }^{4}$ Moderate amounts of weight loss as well as exercise are associated with improvement in insulin sensitivity and sustained improvement in liver enzymes and quality of life. ${ }^{10,26}$ The goal of weight management is to induce a negative calorie balance. This can be accomplished by diet and exercise. Reduction of dietary carbohydrates improve the lipid profile of overweight individuals. Saturated fat intake should also be restricted to prevent diabetes and coronary artery disease. The use of heart healthy diet designed to produce a calorie deficit of 500-1000 calories/day for those who are overweight or obese appears to be rational. Exercise is also proven to be beneficial for coronary artery disease, congestive heart failure, peripheral vascular disease, overall well being among others. Moderate to high-intensity exercise (30 min 3-5 times/week) is ideal for reduction of the risk of co-morbidities associated with obesity. Pharmacological treatment of obesity should be considered in those with a BMI $>30 \mathrm{~kg} / \mathrm{m}^{2}$ or a BMI $>27 \mathrm{~kg} / \mathrm{m}^{2}$ with associated obesity-related comorbidities. There are currently two drugs approved for weight management: (1) Sibutramine and (2) Orlistat. $^{4}$
Insulin sensitising drugs:

It is well established that insulin resistance is a common association with patients with NAFLD and plays an important part in lipid accumulation within the liver and perhaps its progression to NASH. In keeping with this, insulin resistance is predictive of the necroinflammatory form of NAFLD and conditions associated with insulin resistance such as obesity and diabetes are associated with the presence of advanced fibrosis among subjects with NASH. ${ }^{10}$ Given the relationship between insulin resistance and $\mathrm{NASH}$, an insulin sensitizer is preferred in those with diabetes and NASH although there are no published studies to support or refute this concept. ${ }^{4}$ Insulin sensitising drugs such as metformin and the thiozoladinediones such as pioglitazone are being used in phase III clinical trial in patients with NAFLD. ${ }^{10}$

\section{Antioxidants:}

Subjects with NAFLD exhibit increased levels of oxidative stress and lipid peroxidation that may play a part in disease progression. Vitamin E is a potent antioxidant and has been evaluated among paediatric and adult patients with NAFLD. Two small pilot trials have shown reduction of ALT levels among adult and paediatric patients with NASH. ${ }^{10,27}$ But combination therapy with Vit $\mathrm{E}$ and Pioglitazone was found to be significantly better than vitamin $\mathrm{E}$ alone. ${ }^{27}$ It is important to note that in two of the studies, there was at least instance each of hepatotoxicity requiring discontinuation of the drug. ${ }^{4}$

Other hepato-protective agents:

A variety of hepato-protective agents used in other liver disease have been evaluated in patients with NAFLD. Pentoxifylline inhibits TNF and has been shown to improve short term survival in severe alcoholic hepatitis. Early pilot trials have shown improvement in aminotransaminases in NAFLD patients with $1200-1600 \mathrm{mg} /$ daily of pentoxifylline.

Ursodeoxycholic acid (UDCA) has antiinflammatory, immune modulating and antiapoptotic properties and is widely used in chronic cholestatic liver diseases. ${ }^{1}$ In a study a significant improvement in the liver enzymes and degree of steatosis was found at two years of treatment as compared with baseline; this significant improvement in liver 
enzymes and steatosis was also seen in the placebo group. The improvement seen with UDCA treatment was not significantly better than that seen in the placebo group. Based on this study, UDCA is not recommended for the treatment of NAFLD. ${ }^{4,10,}$

Lipid lowering drugs:

As hypertriglyceridaemia and low HDL cholesterol levels are a manifestation of insulin resistance and common among subjects with NAFLD, several investigators have used lipid lowering drugs to treat NAFLD. ${ }^{10}$ A decline in transaminase levels and normalization of ultrasonographic evidence of fatty liver were observed on treatment with omega-3 fatty acids in patients with hypertriglyceridemia, with atorvastatin in those with hypercholesterolemia, and orlistat in overweight patients with hyperlipidemia. ${ }^{28}$

Potential Treatments for Nonalcoholic Fatty Liver Disease $^{4,21}$

Lifestyle-modifications to induce weight loss-

Caloric restriction

Physical activity

$\underline{\text { Insulin-sensitizing agents }}$

Biguanide (metformin)

Thiazolidinediones (rosiglitazone, pioglitazone)

Leptin (not available commercially)

Antioxidants-

Vitamin E

Betaine

Silymarin

Vitamin C

Hepatoprotective agents-

Ursodeoxycholic acid

Lipid-lowering agents-Fibrates such as gemfribrozil, Statins

Anti TNF regimen

Pentoxyfylline

Adiponectin

\section{Conclusion}

Non-alcoholic fatty liver disease is increasingly being recognized as an important and common condition. NASH is not currently considered as a merely benign clinical entity, but is rather thought as a common disease with a variety of clinical sequelae including liver cirrhosis and even hepatocellular carcinoma. It is usually associated with the metabolic syndrome. Its definite diagnosis requires a liver biopsy. The need for a liver biopsy should be individualized and requires consideration of the likelihood of having alternate liver diseases as well as the likelihood of having advanced fibrosis and whether confirmation of the stage of disease will affect management. There is currently no established pharmacologic treatment of NAFLD. Treatment should be focused on correction of the underlying metabolic syndrome. The role of specific pharmacologic treatment continues to evolve. Several large clinical trials using a variety of agents are currently under way and should provide additional treatment option for those with NASH.

\section{References:}

1. Das K, Kar P. Non-alcoholic steatohepatitis. J Assoc Physicians India 2005; 53: 195-9.

2. Choudhury J, Sanyal AJ. Clinical aspects of fatty liver disease. Semin Liver Dis 2004; 24: 349-62.

3- Angulo P. Nonalcoholic fatty liver disease. N Engl J Med 2002; 346:1221-3.

4. Seela Ramesh, Arun J. Sanyal. Evaluation and management of non-alcoholic steatohepatitis. Hepatology 2005; 42: Suppl S2-S12.

5. Ludwig J, Viaggiano TR, McGill DB, Oh BJ. Nonalcoholic steatohepatitis: Mayo Clinic experience with a hitherto unnamed disease. Mayo Clin Proc 1980; 55: 434-438.

6. Giulio Marchesini, Mara Brizi, Giampaolo Bianchi, Sara Tomassetti, Elisabetta Bugianesi et al. Nonalcoholic Fatty Liver Disease- A Feature of the Metabolic Syndrome. Diabetes 2001; 50:1844-1850.

7. Ishii H. Current trend of NASH/NAFLD in Asia-Pacific region. Nippon Rinsho 2006; 64:1017-9.

8. Daniel S, Ben-Menachem T, Vasudevan G, Ma CK, Blumenkehl M. Prospective evaluation of unexplained chronic liver transaminase abnormalities in asymptomatic and symptomatic patients. Am J Gastroenterol 1999; 94: 3010-4.

9. Browning JD, Szczepaniak LS, Dobbins R, Nuremberg P, Horton JD, Cohen JC, Grundy SM, Hobbs HH. Prevalence of hepatic steatosis in an urban population in the United States: impact of ethnicity. Hepatology 2004; 40:1387-95.

10. Adams LA, Angulo P. Treatment of non-alcoholic fatty liver disease. Postgraduate Medical Journal 2006; 82:315-322.

11. Jeffrey D. Browning and Jay D. Horton. Molecular mediators of hepatic steatosis and liver injury. J. Clin. Invest 2004; 114:147-152.

12. Haque M, Sanyal AJ. The metabolic abnormalities associated with non-alcoholic fatty liver disease. Best Pract Res Clin Gastroenterol 2002; 16: 709-31.

13- Pagano, C., Soardo, G., Pilon, C., Milocco, C., Basan, L., Milan, G., Donnini, D., Faggian, D., Mussap, M., Plebani, 
M., Avellini, C., Federspil, G., Sechi, L. A., Vettor, R. Increased Serum Resistin in Nonalcoholic Fatty Liver Disease Is Related to Liver Disease Severity and Not to Insulin Resistance. J Clin Endocrinol Metab 2006; 91: 1081-1086

14. Neuschwander-Tetri BA, Caldwell SH. Nonalcoholic steatohepatitis: summary of an AASLD single topic conference. Hepatology 2003; 37:1202-19.

15. Leon A. Adams, James F. Lymp, Jenny St. Sauver, Schuyler O. Sanderson, Keith D. Lindor, Ariel Feldstein and Paul Angulo The Natural History of Nonalcoholic Fatty Liver Disease: A Population-Based Cohort Study. Gastroenterology 2005; 129: 113-21.

16. Joy D, Thava VR, Scott BB. Diagnosis of fatty liver disease: is biopsy necessary? Eur J Gastroenterol Hepatol 2003; 15: $539-43$.

17. Saadeh S, Younossi ZM, Remer EM, Gramlich T, Ong JP, Hurley M, Mullen KD, Cooper JN, Sheridan MJ. The utility of radiological imaging in nonalcoholic fatty liver disease. Gastroenterology 2002; 123: 745-50.

18. Mottin C.C., Moretto M., Padoin A.V., Swarowsky A.M., Toneto M.G., Glock L., Repetto G. The role of ultrasound in the diagnosis of hepatic steatosis in morbidly obese patients. Obes Surg 2004; 14: 635-7.

19. Hashimoto E. Diagnostic criteria for nonalcoholic steatohepatitis. Nippon Rinsho 2006; 64: 1025-32

20. Angulo P, Keach JC, Batts KP, Lindor KD. Independent predictors of liver fibrosis in patients with nonalcoholic steatohepatitis. Hepatology 1999; 30:1356-62.

21. Jeanne M. Clark, Anna Mae Diehl. Nonalcoholic Fatty Liver Disease. An Under recognized Cause of Cryptogenic Cirrhosis. JAMA 2003; 289: 3000-3004

22. Sanyal AJ, Banas C, Sargeant C, Luketic VA, Sterling RK, Stravitz RT, Shiffman ML, Heuman D, Coterrell A, Fisher RA, Contos MJ, Mills AS. Similarities and differences in outcomes of cirrhosis due to nonalcoholic steatohepatitis and hepatitis C. Hepatology 2006; 43: 682-9

23. Targher G, Bertolini L, Poli F, Rodella S, Scala L, Tessari R, Zenari L, Falezza G. Nonalcoholic Fatty Liver Disease and Risk of Future Cardiovascular Events Among Type 2 Diabetic Patients. Diabetes 2005; 54:3541-3546.

24. Angulo P, Lindor KD. Treatment of nonalcoholic fatty liver: present and emerging therapies. Semin Liver Dis 2001; 21 : 81-8.

25. Reid BM, Sanyal AJ. Evaluation and management of nonalcoholic steatohepatitis. Eur J Gastroenterol Hepatol 2004; 16:1117-22.

26. I J Hickman, J R Jonsson, J B Prins, S Ash, D M Purdie, A D Clouston and E E Powell. Modest weight loss and physical activity in overweight patients with chronic liver disease results in sustained improvements in alanine aminotransferase, fasting insulin, and quality of life. Gut 2004; 53:413-419.

27. Sanyal AJ, Mofrad P, Contos MJ, Sargeant C, Luketic VA, Sterling RK. A pilot study of vitamin $\mathrm{E}$ versus vitamin $\mathrm{E}$ and pioglitazone for the treatment of nonalcoholic steatohepatitis. Clin Gastroenterol Hepatol 2004; 2:1107-15.

28. Hatzitolios A, Savopoulos C, Lazaraki G, Sidiropoulos I, Haritanti P, Lefkopoulos A, Karagiannopoulou G, Tzioufa V, Dimitrios K. Efficacy of omega-3 fatty acids, atorvastatin and orlistat in non-alcoholic fatty liver disease with dyslipidemia. Indian J Gastroenterol 2004; 23:131-134.

29. Sanyal AJ. Review article: non-alcoholic fatty liver disease and hepatitis C--risk factors and clinical implications.Aliment Pharmacol Ther 2005; 22 Suppl 2:48-51.

30. L Rubbia-Brandt, P Fabris, S Paganin, G Leandro, P-J Male, E Giostra, A Carlotto, L Bozzola, A Smedile and F Negro. Steatosis affects chronic hepatitis $\mathrm{C}$ progression in a genotype specific way. Gut 2004; 53: 406-412. 